\title{
Impact of Thin LT-GaN Cap Layers on the Structural and Compositional Quality of MOVPE Grown InGaN Quantum Wells Investigated by TEM
}

\author{
F. Ivaldi, S. Kret, A. Szczepańska \\ Institute of Physics, Polish Academy of Sciences, al. Lotników 32/46, 02-668 Warsaw, Poland \\ R. Czernecki, M. Kryśko, S. Grzanka and M. Leszczyński
}

Institute of High Pressure Physics, Polish Academy of Sciences, Sokołowska 29/37, 01-142 Warsaw, Poland

Two samples containing InGaN quantum wells have been grown by metal-organic vapor phase epitaxy on high pressure grown monocrystalline GaN (0001). Different growth temperatures have been used to grow the wells and the barriers. In one of the samples, a low temperature $\mathrm{GaN}$ layer $\left(730^{\circ} \mathrm{C}\right)$ has been grown on every quantum well before rising the temperature to standard values $\left(900^{\circ} \mathrm{C}\right)$. The samples have been investigated by transmission electron microscopy and X-ray diffraction. Photoluminescence spectra have been measured as well. The influence of the LT-GaN has been investigated in regard to its influence on the structural and compositional quality of the sample.

PACS: 68.35.bg, 68.37.Lp, 68.65.Fg

\section{Introduction}

The attempt to make high quality green light emitting devices on the base of InGaN systems needs relatively high indium concentrations in the quantum wells (QW). At low temperatures, the indium incorporation is most effective. On the other hand, the growth of the quantum barriers (QB) requires higher temperatures. One of the methods tested to achieve this target is the temperature increase after the QWs growth to the optimal temperature for GaN growth. Exposition of the InGaN layers to high temperatures can cause structural changes because of the high temperature instability of InGaN and the related indium desorption process. Understanding the structural properties of MQW grown with these parameters helps optimizing conditions to achieve proper luminescence properties. High In content is required to open the way to new technologies and devices like green lasers and solar cells with high efficiencies. This paper investigates a new method to achieve such a goal and presents a compositional and morphological characterization of InGaN samples grown with different parameters. This can help in understanding the physical processes occuring during growth and leads to the optimization of its conditions.

\section{Experimental}

Two metal-organic vapor phase epitaxy (MOVPE) samples containing each five $2.5 \mathrm{~nm}$ thick InGaN quantum wells have been grown on free standing GaN with different parameters. For sample $B$, the InGaN growth temperature was set to $730^{\circ} \mathrm{C}$, while the $8 \mathrm{~nm}$ thick GaN barriers were deposited at $900{ }^{\circ} \mathrm{C}$. The second sample $C$ - has been grown with a slightly different structure. After the growth of each quantum well, the temperature was kept at $730^{\circ} \mathrm{C}$ and $\mathrm{GaN}$ has been grown for $40 \mathrm{~s}$ $(\approx 1 \mathrm{~nm}$ thickness). Afterwards the temperature has been risen to $900{ }^{\circ} \mathrm{C}$ with a $30 \mathrm{~s}$ ramp and the rest of the barrier has been grown for 3 min.

Cross-sections of all samples have been prepared for transmission electron microscopy (TEM). By polishing and dimpling the samples' region of interest has been thinned to $40 \mu \mathrm{m}$. In the last step of the preparation a Precision Ion Polishing System (PIPS) in double mode has been used to ion mill the specimen. The ion milling took place under nitrogen cooling to reduce the sample's temperature during the process and achieve a more gentle surface removal and less radiation damage. We believe that the sample holder is cooled to about $-120^{\circ} \mathrm{C}$ [1]. The milling was then terminated at low voltage to smooth the specimen.

TEM investigations of all samples has been carried out with a JEOL 2000EX microscope equipped with a LaB6 electron gun operating at $200 \mathrm{kV}$. The samples have been investigated in regard to the morphology, strain and composition of the InGaN QWs. All cross-sectional observations were performed in the $11 \overline{2} 0$ zone axis.

Bright and dark field images of 002 lattice fringes, useful for quantitative analysis, were obtained with a samples' tilting of 3-4 degrees around the [001] direction away 
from the exact pole position. This condition is analogue to the one used in [2], where a more detailed description of the conditions needed for the investigation is given. The evaluation of the local chemical composition was achieved by measurement of the local interplanar 0002 spacing and the use of Vegard's law for InN/GaN compounds. Thin foil relaxation is taken into account with the help of field electron microscopy (FEM) calculations. This image processing technique is based on the principles described in [3].

\section{One- and two-temperature processes}

In a previous work [4] we reported the effects of a two-temperature process on InGaN/GaN QWs with $4.5 \mathrm{~nm}$ nominal thickness (sample A). A degradation causing a reduction of the thickness of the InGaN layers due to indium desorption was observed. This created a stepped QW/QB interface, whose morphology was strongly anisotropic and showed stripe-like structures, which differed in orientation in a micrometer scale. We demonstrated that the bottom and top QW interfaces are rough and the height can drop to 1 monolayer, observing that QWs can locally disappear or have very low indium concentration, as low as 5 at.\%.

These structural inhomogeneities can have a strong impact on the luminescence properties of the specimen as reported in [5], where 3D atom probe has been used to investigate MOVPE grown multiquantum well (MQW) structures. The carrier localization on one side and the spreading of height of the photoluminescence (PL) peaks on the other can be consequences of the morphology of the QW interfaces.

\section{LT-GaN protective layer}

To avoid indium desorption, sample $\mathrm{C}$ has been grown with a protective low temperature GaN layer above every QW. This layer was expected to protect the QW and impeding the occurrence of desorption. A detailed comparison of the geometrical and compositional character of the samples arising from our analysis is given in Table.

Statistics on the four sample investigated.

TABLE

\begin{tabular}{l|c|c|c}
\hline \hline & $A$ & $B$ & $C$ \\
\hline temperature [ $\left.{ }^{\circ} \mathrm{C}\right]$ & $730 / 900$ & $730 / 900$ & $730 / 730 / 900$ \\
stripes width [nm] & $70-186$ & $22-67$ & continuous \\
QW height [nm] & $0.8-2.3$ & $1.0-4.4$ & $2.0-3.5$ \\
interruptions' length [nm] & no & $8-45$ & no \\
period [nm] & 12.3 & 18 & 17.6 \\
indium content [at.\%] & $12-22$ & $13-20$ & $15-20$
\end{tabular}

Figure 1 shows the direct comparison of the active regions of $B$ and $C$ highlighting the differences in the geometry and composition of the MQWs. In sample $B$ the QWs are mostly discontinuous with areas of high indium concentration followed by areas where the indium percentage is very low or zero. As demonstrated in [4], where a plane view of sample $A$ is shown, the inhomogeneities are in fact stripe like InGaN features with a finite width. In the cross-section the stripes appear as InGaN islands (Fig. 1a). The measured average values for these islands' height and period are, respectively, $2.7 \mathrm{~nm}$ and $18 \mathrm{~nm}$. They differ from the nominal values $2.5 \mathrm{~nm}$ and $10.5 \mathrm{~nm}$. Furthermore, it can be observed that the bottom GaN/ InGaN interface is well defined, while the upper interface appears very rough. The estimated indium concentration in the islands (measured in the center of the features) is strongly fluctuating from island to island from $13 \%$ to $20 \%$.
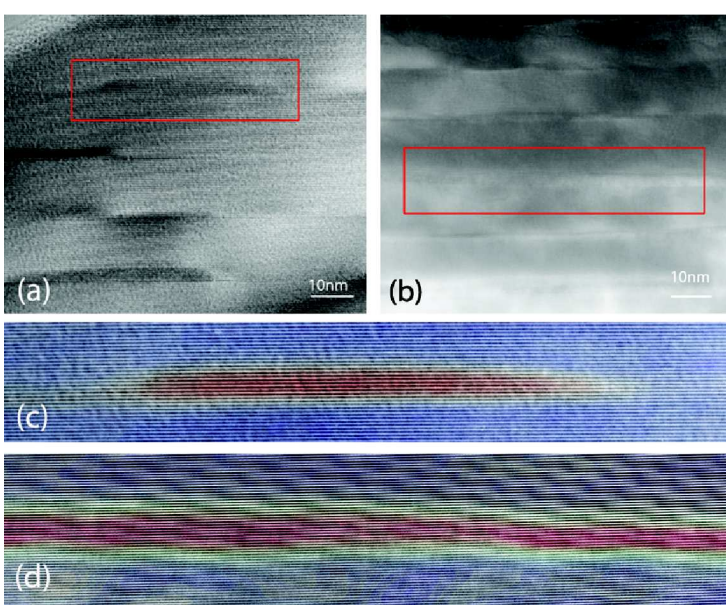

Fig. 1. (a) TEM lattice fringes image of sample $B$. InGaN islands can be observed. (b) TEM image of sample $C$. The QWs are continuous but fluctuating in height. (c) and (d) Color coded indium content map overlayed on the original TEM 002 lattice fringes image. Part (c) refers to the detail in (a) and (d) to the frame in (b).

Sample $C$ - grown with protective LT-GaN layers on top of every well - shows significant differences in comparison to the previously presented samples. The geometry of the QWs is more homogeneous and without interruptions (Fig. 1b). However, the indium concentration varies from well to well and takes values between $13 \%$ and $20 \%$ with rare peaks at $25 \%$.

The QBs' height varies significantly from $11 \mathrm{~nm}$ to $18 \mathrm{~nm}$ and the period of the QWs could be estimated to be $17.6 \mathrm{~nm}$ far beyond the nominal $10.5 \mathrm{~nm}$.

Furthermore, it should be noticed that the LT-GaN layers do not show any defect or a lower crystallographic quality although the growth has been performed at low temperatures.

PL investigations of both samples $-B$ and $C-$ show a green shift of the measured peak for the sample with the protective layers (Fig. 2). The emission wavelength maximum shifts from $480 \mathrm{~nm}$ to $510 \mathrm{~nm}$. This fact seems coherent with the higher indium content determined in sample $C$. The intensity measured for such sample is 
higher in comparison to $B$. This fact can be explained by the absence of interruptions in the wells' geometry and by the higher average In content.

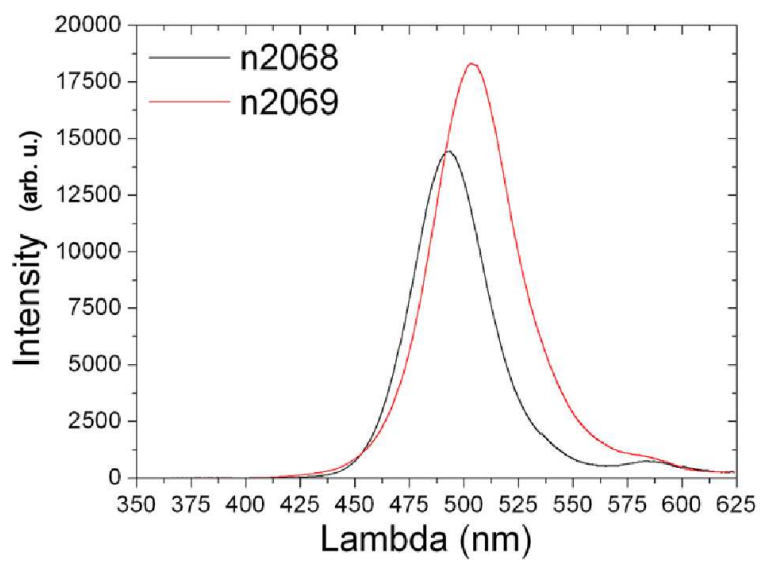

Fig. 2. PL measurements comparing the emission spectra of samples $B$ and $C$. The sample containing LT-GaN layers has a green-shifted spectrum (red).

X-ray diffraction (XRD) measurements shown in Fig. 3 are substantially similar for the sample with and without protective layers. However a difference between the two spectra can be observed. The red curve shows a worse periodicity of the wells/barriers superlattice.

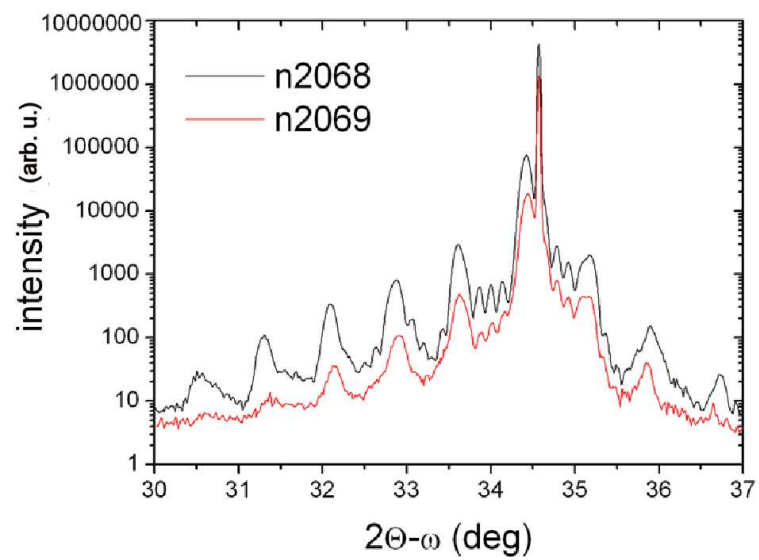

Fig. 3. XRD measurements for sample $B$ and $C$.

The reason for this effect, shown in Fig. 4, could be determined during TEM observation. The QWs are subject to fluctuations, which are increasing progressively from the first to the last grown QW. As a result the QWs partly do not run parallel to the intended growth direction and the barriers' thickness oscillates dramatically between $11 \mathrm{~nm}$ and $20 \mathrm{~nm}$. However the QWs thickness stays constant with few fluctuations and an average of $2.9 \mathrm{~nm}$. We assume that the uncontrolled growth rate of LT-GaN leads to local differences in the growth speed having as a consequence the formation of a stepped growth front. This influences the thickness of the QBs

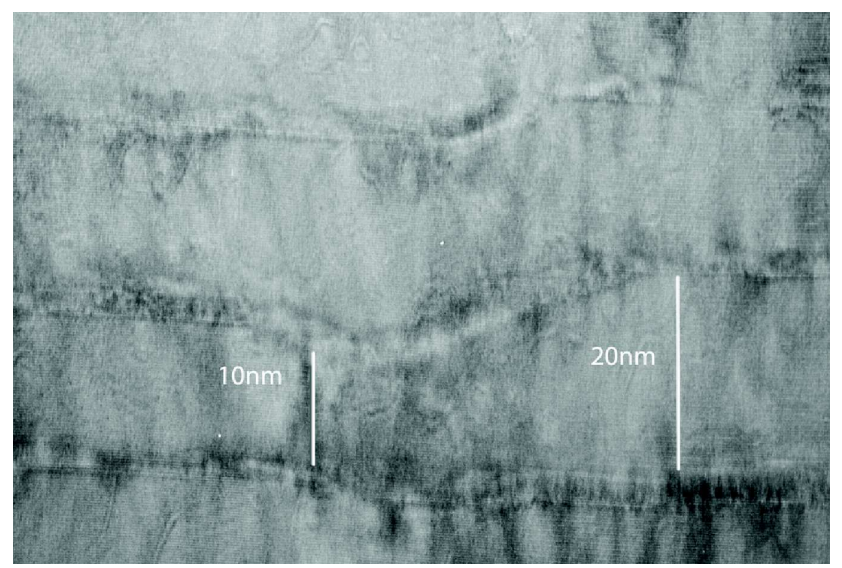

Fig. 4. TEM image of sample $C$ showing relevant variations in the QBs thickness.

which changes locally and results in a worse XRD curve in respect of sample $B$.

\section{Conclusions}

We demonstrated the impact of a thin LT-GaN layer on the structure and composition of MOVPE InGaN QWs. We observed a positive effect of the protective layer on the In distribution in the QWs, preventing desorption. PL measurements show a green shift of the emission peak from $480 \mathrm{~nm}$ to $510 \mathrm{~nm}$. However, XRD and TEM investigation of the samples showed relevant structural issues along the QWs. A negative influence of the protective layer causing steps or valleys along the length of the wells could be observed. These influence the periodicity of the barrier/well superlattice and create unwanted fluctuations along the sample.

\section{Acknowledgments}

Part of this work was supported by the EU under project ITN RAINBOW, contract $\mathrm{N}^{\circ}$ : PITN-GA-2008-213238 and by grant no. 337/N-VIPP/2008/0 of the Polish Ministry of Science and Higher Education.

\section{References}

[1] Gatan Inc. - Manual: Precision Ion Polishing System: Cold Stage Upgrade, 2008.

[2] S. Kret, P. Dluzewski, A. Szczepanska, M. Zak, R. Czernecki, M. Krysko, M. Leszczynski, G. Maciejewski, Nanotechnology 18, 465707 (2007).

[3] S. Kret, P. Ruterana, D. Gerthseni, Phys. Status Solidi B 227, 247 (2001).

[4] S. Kret, F. Ivaldi, K. Sobczak, R. Czernecki, M. Leszczynski, Phys. Status Solidi A 207, 1101 (2010).

[5] R.A. Oliver, M.J. Kappers, C.J. Humphreysi, Phys. Status Solidi C 5, 1475 (2008). 